\title{
Siete psicoterapias probaron ser igualmente efectivas en el tratamiento de la depresión
}

Seven psychotherapies proved equally effectiveness in the treatment of depression

\section{Objetivos}

Comparar la eficacia de siete psicoterapias en pacientes con depresión.

\section{Fuentes de datos}

Publicaciones en PubMed, PsycINFO, Embase, Cochrane Central Register of Controlled Trials y WHO Afro Librar, desde 1966 hasta 2012

\section{Selección de estudios}

Fueron incluidos ensayos clínicos controlados y aleatorizados en los que habían participado pacientes con diagnóstico presuntivo o definitivo de depresión, y que hubieran comparado un tipo de psicoterapia con otro, o bien con otra modalidad controlada (cuidado usual, placebo, lista de espera). Fueron exclui-
Barth J y col. PLoS Med. 2013; 10(5): e1001454. dos los estudios que habían combinado fármacos con psicoterapias o los habían comparado con ellas.

\section{Extracción de datos}

Los estudios fueron evaluados por dos revisores independientes que los clasificaron de acuerdo al tipo de psicoterapia al tamaño muestral (menos de 25 pacientes: pequeño, 25 a 50 pacientes: mediano, más de 50: grande) y la calidad de estudios (determinada por una adecuada ocultación de la asignación, evaluación de resultados y análisis por intención de tratar). El resultado principal evaluado fue la reducción de síntomas depresivos medido por diferentes escalas (Beck Hamilton, etc.)

\section{Resultados principales}

Se resumen en la tabla y el gráfico 1.

Tabla 1. medida de efecto "d" de Cohen*. Comparación por diferencia de medias (IC95\%).

\begin{tabular}{|c|c|c|c|c|c|c|}
\hline & Consejeria & Terapia psicodinámica & $\begin{array}{l}\text { Entrenamiento en } \\
\text { habilidades sociales }\end{array}$ & $\begin{array}{l}\text { Terapia de resolución } \\
\text { de problemas }\end{array}$ & $\begin{array}{c}\text { Terapia } \\
\text { cognitivo-conductual }\end{array}$ & $\begin{array}{l}\text { Terapia de activación } \\
\text { conductual }\end{array}$ \\
\hline Terapia psicodinámica & $\begin{array}{c}-0.1 \\
(-0.41 \text { a } 0.20) \\
\end{array}$ & & & & & \\
\hline $\begin{array}{l}\text { Entrenamiento en } \\
\text { habilidades sociales }\end{array}$ & $\begin{array}{c}-0.01 \\
(-0.60 \text { a } 0.54) \\
\end{array}$ & $\begin{array}{c}0.09 \\
(-0.49 \text { a } 0.20) \\
\end{array}$ & & & & \\
\hline $\begin{array}{l}\text { Terapia de resolución } \\
\text { de problemas }\end{array}$ & $\begin{array}{c}-0.12 \\
(-0.36 \text { a } 0.13) \\
\end{array}$ & $\begin{array}{c}-0.02 \\
(-0.35 \text { a } 0.32) \\
\end{array}$ & $\begin{array}{c}-0.11 \\
(-0.68 \text { a } 0.49) \\
\end{array}$ & & & \\
\hline $\begin{array}{c}\text { Terapia } \\
\text { cognitivo-conductual }\end{array}$ & $\begin{array}{c}-0.16 \\
(-0.33 \text { a } 0.01) \\
\end{array}$ & $\begin{array}{c}-0.06 \\
(-0.33 \text { a } 0.22) \\
\end{array}$ & $\begin{array}{c}-0.15 \\
(-0.67 \text { a } 0.41) \\
\end{array}$ & $\begin{array}{c}-0.04 \\
(-0.25 \text { a } 0.17)\end{array}$ & & \\
\hline $\begin{array}{l}\text { Terapia de activación } \\
\text { conductual }\end{array}$ & $\begin{array}{c}-0.18 \\
(-0.47 \text { a } 0.12)\end{array}$ & $\begin{array}{c}-0.08 \\
(-0.43 \text { a } 0.26)\end{array}$ & $\begin{array}{c}-0.17 \\
(-0.72 \text { a } 0.43) \\
\end{array}$ & $\begin{array}{c}-0.06 \\
(-0.38 \text { a } 0.26) \\
\end{array}$ & $\begin{array}{c}-0.02 \\
(-0.29 \text { a } 0.25) \\
\end{array}$ & \\
\hline Terapia interpersonal & $\begin{array}{c}-0.3 \\
(-0.54 a-0.55)\end{array}$ & $\begin{array}{c}-0.19 \\
(-0.53 \text { a } 0.14)\end{array}$ & $\begin{array}{c}-0.29 \\
(-0.85 \text { a } 0.31) \\
\end{array}$ & $\begin{array}{c}-0.18 \\
(-0.46 \text { a } 0.09)\end{array}$ & $\begin{array}{c}-0.14 \\
(-0.33 \text { a } 0.07)\end{array}$ & $\begin{array}{c}-0.12 \\
(-0.44 \text { a } 0.20)\end{array}$ \\
\hline
\end{tabular}

Gráfico 1: medida de efecto "d" de Cohen* (Forest Plot).

\section{Conclusiones}

Todas las psicoterapias evaluadas mostraron tener un efecto beneficioso en la reducción de síntomas en pacientes con depresión, con una eficacia similar.

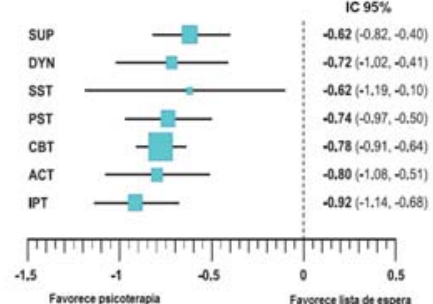

\section{Comentario}

Los meta-análisis en red* permiten realizar comparaciones indirectas entre intervenciones que no fueron comparadas "cabeza a cabeza" en un ensayo clínico individual'. Ante este diseño de investigación, es importante ser cauteloso en la interpretación de los resultados, que debe quedar supeditada a la cantidad de pacientes en cada rama terapéutica, al número de conexiones en la red, a la heterogeneidad conceptual y a la coherencia estadística. Consideramos que este trabajo supera estas li-mitaciones para la mayoría de las comparaciones. Sus principales limitaciones son el sesgo de publicación que fue identificado a través de asimetría de la distribución de los resul- tados en el test de Egger* y la existencia de una poca cantidad de estudios de buena calidad que hayan evaluado algunas de las psicoterapias. Vale destacar que esta revisión sistemática no incluyó investigaciones que hubieran evaluado la recaída y la recurrencia del trastorno depresivo².

\section{Conclusiones del comentador}

Estos resultados nos permiten elegir entre una amplia gama de opciones terapéuticas, que pueden ser ofrecidas de acuerdo a las características del paciente y a la disponibilidad del recurso humano formado para implementarla ${ }^{3}$

Juan Víctor Ariel Franco [ Servicio de Medicina Familiar y Comunitaria del Hospital Italiano de Buenos Aires, juan.franco@ @ospitalitaliano.org.ar] Franco J. Siete psicoterapias probaron ser igualmente efectivas en el tratamiento de la depresión. Evid Act Pract Ambul. Ene-Mar 2014;17(1):14. Comentado de: Barth J. Comparative efficacy of seven psychotherapeutic interventions for patients with depression: a network meta-analysis. PLoS Med. 2013; 10(5): e1001454.

\section{Referencias}

1. Mills E y col. Demystifying trial networks and network meta-analysis. BMJ. 2013 May 14; 346:f2914.

2. Paykel E. Partial remission, residual symptoms, and relapse in depression. Dialogues Clin Neurosci. 2008; 10(4):431-7.

3. Simon G y col. Personalized medicine for depression: can we match patients with treatments? Am J Psychiatry. 2010 Dec; 167(12):1445-55. doi: 10.1176/appi.ajp.2010.09111680. Epub 2010 Sep 15. 